\title{
Comparison of chemical composition, antioxidant and antimicrobial potentials of essential oils and oleoresins obtained from seeds of brassica juncea and sinapis Alba
}

\begin{abstract}
Many consumers are demanding foods without what they perceive as artificial and harmful chemicals, including many used as antimicrobials and preservatives in food. Consequently, interest in more natural, antimicrobials as potential alternatives to conventional additives to extend shelf life and combat foodborne pathogens has heightened. In the present study, phytochemical, in vitro antioxidant and antimicrobial potentials of essential oils and oleoresins of Brassica juncea and Sinapis Alba seeds were determined qualitatively. Presence of allyl isothiocyanates, alkyl isothiocyantes in essential oils and oleoresins was confirmed by using gas chromatography (GC) and $\mathrm{GC}$ coupled to mass spectrometry (GC-MS). Antioxidant effectiveness was examined by five different methods, namely the ferric thiocyanate (FTC) method, the 2,2'-diphenyl-1-picrylhydrazyl (DPPH) radical scavenging method, determination of the metal chelating power, and determination of the peroxide and thiobarbituric acid values in mustard oil at $0.02 \%$ concentration. Food poisoned, inverted Petri plate, agar well diffusion and disk diffusion methods were employed for investigating antimicrobial potentials. Such results indicate that essential oils of B.juncea and $S$. alba can be possible candidates for further investigations to isolate and characterize their active principles as possible new natural preservatives.
\end{abstract}

Keywords: in vitro antioxidant, essential oils, oleoresins, food poisoned, inverted petriplate
Volume 4 Issue 4 - 2017

\author{
Sunita Singh,' SS Das,' G Singh,' Marina \\ Perroti,' Carola Schuff,' César AN Catalán ${ }^{2}$ \\ 'Chemistry Department, Deen Dayal Upadhyaya Gorakhpur \\ University, India \\ ${ }^{2}$ Facultad de Bioquímica Química y Farmacia, Universidad \\ Nacional de Tucumán, Argentina
}

Correspondence: Sunita Singh, Chemistry Department, DDU Gorakhpur University Gorakhpur-273009, Uttarpradesh, India, Email oceans.singh@gmail.com

Received: October 26, 2016 | Published: June 26, 2017
Abbreviations: GC, gas chromatography; GC-MS, GC coupled to mass spectrometry; FTC, ferric thiocyanate; DDPH, 2,2'-diphenyl1-picrylhydrazyl; BJEO, B.juncea; SANH, S.alba N-hexane; TBA, thiobarbituric acid; EDT, ethylene diamine tetraacetic acid; PG, propyl gallate; MTCC, microbial type culture collection; AITC, allyl isothiocyanate

\section{Introduction}

Recently there has been a renewed interest in improving health and fitness through the use of more natural products. Herbs and spices have been used for thousands of years to enhance the flavor, color and aroma of food, also known for their preservative and medicinal value. Recent reports suggest that cruciferous vegetables act as a good source of natural antioxidants due to the high levels of carotenoids, tocopherols and ascorbic acid. In addition to carotenoids, tocopherols, and ascorbic acid, most of the antioxidative effect related to plant food intake is mainly due to the presence of phenolic compounds, which have been associated with flavour and colour characteristics of fruits and vegetables. In this aspect, the popularity and consumption of vegetable Brassica species is increasing because of their nutritional value. Brassica foods are very nutritive, providing nutrients and health-promoting Phytochemicals such as vitamins, carotenoids, fiber, soluble sugars, minerals, glucosinolates and phenolic compounds. Brassica is one of the most ancient spices. It has 3 varieties namely black, brown and white/yellow. Brassica juncea (Brown mustard) is largely cultivated. Brown mustard plant produces tiny yellow colored flowers, which almost cover the plant. The plant is extensively grown for its mustard and as fodder crop. In addition, this species is known to be of great medicinal importance due to its antineoplastic, antimicrobial, and insecticidal activities. The plant is a folk remedy for arthritis, foot ache, lumbago, and rheumatism. Sinapis alba is an economically important plant of Brassicaceae, commonly known as yellow or white mustard, and growing well in hot and dry environments. ${ }^{2}$ However, there is limited published research on essential oil composition and activity of both species in spite of the historical and traditional knowledge of both oils' medicinal importance. The popularity and consumption of vegetable Brassica species is increasing because of their nutritional value. Brassica crops have been related to the reduction of the risk of chronic diseases including cardiovascular diseases and cancer. Brassica foods are very nutritive, providing nutrients and health-promoting Phytochemicals such as vitamins, carotenoids, fiber, soluble sugars, minerals, glucosinolates and phenolic compounds. ${ }^{3}$

\section{Materials and methods}

Brown and yellow mustard seeds were purchased from local market of Gorakhpur. The fresh and mature berries of B.juncea and $S$. alba were washed; sun dried and pulverized into a fine powder. $150 \mathrm{~g}$ of spice powder was subjected to hydrodistillation in a Clevenger's type apparatus for $6 \mathrm{~h}$ according to method recommended by European Pharmacopoeia ${ }^{4}$ to obtain B.juncea (BJEO) and S.alba (SAEO) essential oils. $30 \mathrm{~g}$ of spice was loaded on the Soxhlet's apparatus and extracted with the solvent for $3 \mathrm{~h}$ to obtain an oleoresin. After complete extraction, the solvents were distilled off to obtain viscous B.juncea ethanol (BJET), B.juncea n-hexane (BJNH), S.alba ethanol (SAET), and $S . a l b a$ n-hexane (SANH) oleoresins which were stored at $4 \pm 1^{\circ} \mathrm{C}$. 


\section{Reagents}

Thiobarbituric acid (TBA), 2,2'-diphenyl-1-picrylhydrazyl radical (DPPH) and linoleic acid are of Across (New Jersey, USA); BHT, BHA, ethylene diamine tetraacetic acid (EDTA) and propyl gallate (PG) are of sd fine chemicals Ltd. Mumbai, India, Tween 20 and Ferrozine (Merck Pvt. Ltd. Mumbai, India) were used for antioxidant assays. Ampicillin was purchased from Ranbaxy fine chemicals (New Delhi) India. Crude mustard oil was obtained from local oil mill in Gorakhpur. All solvents used were of analytical grade.

\section{Phytochemistry}

The extracted essential oils and oleoresins were subjected to the Gas Chromatography (GC) and GC-Mass Spectroscopy (GC-MS) for analysis using Hewlett-Packard gas chromatograph (Model-6890) coupled with a Quadruple Mass Spectrometer 5973 and a Perkins Elmer Elite-5MS capillary column [5\% phenyl methyl siloxane; $(30 \mathrm{~m} \times 0.25 \mathrm{~mm} \times 0.25 \mu \mathrm{m})]$. For the oil, oven temperature was programmed as: at $60^{\circ} \mathrm{C}$ for one min; then increased from 60 to $185^{\circ} \mathrm{C}$ at the rate of $1.5^{\circ} \mathrm{C} / \mathrm{min}$ and held at $185 \mathrm{C}$ for one min; then again increased from $185^{\circ} \mathrm{C}$ to $275^{\circ} \mathrm{C}$ at the rate of $9^{\circ} \mathrm{C} / \mathrm{min}$ and held at $275^{\circ} \mathrm{C}$ for two min. The oven temperature for oleoresins was programmed as follows: $80^{\circ} \mathrm{C}$ (zero min), increased from $80^{\circ} \mathrm{C}$ to $300^{\circ} \mathrm{C}$ at the rate of $3.5 \mathrm{C} / \mathrm{min}$ and held at $300^{\circ} \mathrm{C}$ for $5 \mathrm{~min}$. The components were identified on the basis of comparison of their retention indices and mass spectra with published data ${ }^{5}$ and computer matching was done with the WILEY 275 and National Institute of Standards Technology (NIST 3.0) libraries provided with the computer controlling GC-MS systems.

\section{Antioxidant assays}

Sample preparation: The essential oils and oleoresins were added individually to unrefined crude linseed oil at the concentration of $200 \mathrm{ppm}(\mathrm{v} / \mathrm{v})$. Synthetic antioxidants such as BHA, BHT and PG were also added to mustard oil at the same concentration, i.e. 200ppm $(\mathrm{v} / \mathrm{v})$. A control sample was prepared under similar condition without any additive. They were subjected to the Schaal oven test ${ }^{6}$ in $100 \mathrm{~mL}$ open beakers at $60^{\circ} \mathrm{C}$.

Lipid peroxidation assays: The antioxidant activity of essential oils and oleoresins in the oxidation of mustard oil was examined by comparing the activity of known antioxidants such as BHA, BHT and PG by the methods reported by Singh et al. ${ }^{7}$ for peroxide and TBA values.

Complementary antioxidant assays: Further determination of antioxidant activity of essential oils and oleoresins were determined by the methods reported earlier by Singh et al. ${ }^{8}$ namely; ferric thiocyanate method, metal chelating activity and radical scavenging activity on DPPH.

\section{Antimicrobial investigations}

All the fungal and bacterial strains for antimicrobial investigations were procured from Microbial Type Culture Collection (MTCC), Institute of Microbial Technology, Chandigarh, India.

Antifungal investigations: In order to determine the antifungal efficacy of the volatile oil and oleoresins, the pathogenic fungus Aspergillus niger (AN;1884), Aspergillus flavus (AF;2479), Fusarium monoliforme (FM;1893), Fusarium graminearum (FG;2088) and Penicillium viridicatum $(P V ; 2007)$ were undertaken. Cultures of each of the fungi were maintained on Czapek dox agar media with adjusting $p \mathrm{H}$ 6.0-6.5 and slants were stored at $4{ }^{\circ} \mathrm{C}$. The antifungal activity of the volatile oil and oleoresins against fungi were undertaken using inverted petriplate ${ }^{9}$ and food poisoned techniques. ${ }^{10}$

Antibacterial investigations: Four pathogenic bacteria Bacillus subtilis [BS;1790], Staphylococcus aureus [SA;3103] (gram positive), Escherichia coli [EC;1672], Pseudomonas aeruginosa [PA; 1942] (gram negative) were selected for present study. The agar well diffusion method $^{10}$ was employed for the determination of antibacterial activity. Briefly, a suspension of the test microorganism $(0.1 \mathrm{ml})$ was spread on a previously prepared, dried nutrient agar plate by using a sterile bent rod. The wells were $10 \mathrm{~mm}$ in diameter cut from the agar and different concentration of essential oils or extracts $(5$ and $10 \mu 1$ diluted in $1 \mathrm{~mL}$ dimethyl sulphoxide) were delivered into them. The control plate without the addition of essential oil or extract containing DMSO was also maintained under the same conditions. After incubating for $24 \mathrm{~h}$ at $37^{\circ} \mathrm{C}$, all plates were examined for any zones of growth inhibition (in $\mathrm{mm}$ ).

\section{Statistical analyses}

Experimental results were the means \pm standard deviation of three parallel measurements (data are not shown). Significant differences between means were determined by Student's $t$-test by using a Microsoft Excel (Microsoft Office, India) statistical analysis program and $p<0.05$ was considered as significant. Standard deviation bars has been provided in the figures.

\section{Results and discussions}

\section{Phytochemistry}

A detailed and careful interpretation of the experimental data (EM fragmentation and retention indices) obtained from GC-MS analysis resulted in the identification of a large number of components in the essential oils and oleoresins of B. juncea and S. alba (Table $1 \& 2$ ). Table $1 \& 2$ shows identification of 8 and 5 components in BJEO and SAEO representing about $96.4 \%$ and $98.5 \%$ respectively of the total amount. Table $1 \& 2$ also shows the chemical composition of ethanol and n-hexane oleoresins extracted from B.juncea and S.alba. A total of 13 and 20 components were identified in BJET and BJNH representing about $62.1 \%$ and $78.6 \%$ respectively of the total amount whereas in SAET and SANH, a total of 13 and 16 components were identified representing about $63.7 \%$ and $77.2 \%$ respectively of the total amount. The major components in essential oils obtained from $B$. juncea and $S$. alba were allyl isothiocyanate, 1-butene-4-thiocyanate along with minor traces of thymol, 2-phenylethyl-isothiocyante. The oleoresin extracted in ethanol and n-hexane for $B$.juncea and $S$. alba contains 5-hydroxymethylfurfural, palmitic acid, oleic acid and stigmast-5-en$\beta$-ol. Presence of allyl isothiocyanates, alkyl isothiocyantes in essential oils and oleoresins may be the results of hydrolysis of glucosinates. ${ }^{11}$ These decomposition products are volatile and also play an important role in the characteristic aroma or off-odor of Brassicacae responsible for the pungent smell of the oil. The presence of oleic acid, linoleic acid, palmitic acid was well supported by the work of Joardar et al. ${ }^{12}$

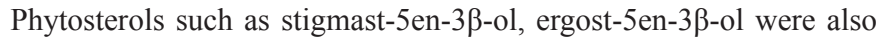
reported by some workers.

\section{Antioxidant investigations}

Lipid peroxidation assays: For the assessment of antioxidant activity of $B$. juncea and $S$. alba essential oils and oleoresins, crude linseed oil 
having initial peroxide value $4.0 \mathrm{meq} \mathrm{kg}^{-1}$ was selected. During this period, the peroxide value of the control increases to $221 \mathrm{meq} \mathrm{kg}^{-1}$.

Table I Brassica juncea essential oil and oleoresins composition

\begin{tabular}{|c|c|c|c|c|c|}
\hline Compounds & BJEO \% MS* & BJET \% MS** & BJNH \% MS** & RI\# & identification $^{\Phi}$ \\
\hline Allylisothiocyanate & 73.4 & --- & --- & 890 & MS, RI \\
\hline butane-1-isothiocyanate & $\operatorname{tr}$ & ---- & ---- & 943 & MS, RI \\
\hline 1-butene-4-isothiocyanate & 19.1 & --- & 0.5 & 978 & MS, RI \\
\hline 5-hydroxymethyl furfural & ---- & 7.7 & 0.5 & 1020 & MS, RI \\
\hline p-cymene & 0.3 & --- & --- & 1019 & MS, RI, co-GC \\
\hline Limonene & $\operatorname{tr}$ & --- & ---- & 1024 & MS, RI, co-GC \\
\hline 1,8-cineole & 0.3 & ---- & ---- & 1026 & MS, RI, co-GC \\
\hline Thymol & 1.4 & 0.6 & 0.3 & 1292 & MS, RI, co-GC \\
\hline E,E-2,4-decadienal & ---- & ---- & 0.2 & 1314 & MS, RI \\
\hline 3-methylthiopropyl-isothiocyanate & 0.7 & --- & --- & 1318 & MS \\
\hline benzyl-isothiocyanate & $\operatorname{tr}$ & --- & ---- & 1361 & MS \\
\hline$\beta$-caryophyllene & $\operatorname{tr}$ & ---- & ---- & 1415 & MS, RI, co-GC \\
\hline 2-phenylethyl-isothiocyanate & 1.2 & ---- & ---- & 1465 & MS, RI \\
\hline myristic acid & ---- & 0.5 & 0.3 & 1739 & MS, RI, co-GC \\
\hline palmitic acid & ---- & 13.4 & 12 & 1967 & MS, RI, co-GC \\
\hline ethyl palmitate & ---- & 0.3 & --- & 1980 & MS, RI, co-GC \\
\hline linoleic acid & ---- & 3 & 6.4 & 2124 & MS, RI, co-GC \\
\hline oleic acid & ---- & 19.3 & 14 & 2128 & MS, RI, co-GC \\
\hline ethyl linoleate & ---- & ---- & ---- & 2141 & MS, RI, co-GC \\
\hline ethyl oleate & ---- & ---- & ---- & 2145 & MS, RI, co-GC \\
\hline stearic acid & ---- & 2 & 2 & 2157 & MS, RI, co-GC \\
\hline Monopalmitin & ---- & 1.3 & ---- & --- & MS \\
\hline Heptacosane & ---- & ---- & 0.7 & ---- & MS \\
\hline Octacosane & ---- & ---- & 1.2 & ---- & MS \\
\hline Nonacosane & ---- & ---- & 2.4 & ---- & MS \\
\hline Triacontane & ---- & ---- & 2.4 & --- & MS \\
\hline$\gamma-\tau \mathrm{o} \chi \mathrm{o} \pi \eta \varepsilon \mathrm{\alpha} \lambda$ & --- & 1 & 0.9 & --- & MS \\
\hline Hentriacontane & ---- & ---- & 3.8 & --- & MS \\
\hline vitamin $\mathrm{E}$ & ---- & ---- & 0.3 & --- & MS \\
\hline ergosta-5,22-dien-3-ol & ---- & 2.4 & 5.2 & --- & MS \\
\hline 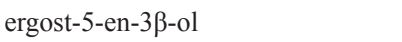 & ---- & 2.7 & 5.7 & --- & MS \\
\hline Dotriacontane & --- & --- & 2.3 & --- & MS \\
\hline stigmast-5-en-3 $\beta$-ol & ---- & 7.9 & 17.5 & --- & MS \\
\hline Total & 96.4 & 62.1 & 78.6 & & \\
\hline
\end{tabular}

BJEO: Brassica juncea essential oil

BJET- Brassica juncea ethanol oleoresin

$\mathrm{BJNH}$ - Brassica juncea $\mathrm{n}$-hexane oleoresin

tr: trace $<0.05$

\#:The retention index was calculated using a homologous series of n-alkanes C8-C22;

$\Phi$ Co-GC: co-injection with an authentic sample. Percentages are

the mean of three runs and were obtained from electronic integration measurements using selective mass detector. 
Table 2 Composition of essential oils and oleoresins obtained from Sinapis alba

\begin{tabular}{|c|c|c|c|c|c|}
\hline Compounds & SAEO \% MS & SAET $\%$ MS & SANH \% MS & RI\# & Identification $\Phi$ \\
\hline Allylisothiocyanate & 0.4 & ---- & ---- & 890 & MS, RI \\
\hline butane-1-isothiocyanate & 0.4 & ---- & ---- & 943 & MS, RI \\
\hline 1-butene-4-isothiocyanate & 96.9 & --- & --- & 978 & MS, RI \\
\hline 5-hydroxymethyl furfural & --- & 21 & --- & 1020 & MS, RI \\
\hline 2-phenylethyl-isothiocyanate & 0.8 & $\operatorname{Tr}$ & --- & 1465 & MS, RI \\
\hline palmitic acid & --- & 4.5 & 3.1 & 1967 & MS, RI, co-GC \\
\hline linoleic acid & --- & 9.6 & 3.2 & 2124 & MS, RI, co-GC \\
\hline oleic acid & ---- & 6.4 & 7.8 & 2128 & MS, RI, co-GC \\
\hline ethyl linoleate & ---- & ---- & 1.6 & 2141 & MS, RI, co-GC \\
\hline ethyl oleate & ---- & ---- & 1.8 & 2145 & MS, RI, co-GC \\
\hline stearic acid & ---- & 2.2 & --- & 2157 & MS, RI, co-GC \\
\hline monopalmitin & --- & 2.7 & $\operatorname{tr}$ & --- & MS \\
\hline Octacosane & --- & --- & 1.2 & 2800 & MS, RI, co-GC \\
\hline nonacosane & --- & --- & 2.2 & 2900 & MS, RI, co-GC \\
\hline Triacontane & --- & --- & 1.8 & 3000 & MS, RI, co-GC \\
\hline$\gamma-\tau \circ \chi о \pi \eta \rho о \lambda$ & --- & 0.7 & 1.3 & 3049 & MS \\
\hline hentriacontane & ---- & $\operatorname{Tr}$ & 3 & 3100 & MS \\
\hline vitamin E & ---- & 0.6 & 1.7 & ---- & MS \\
\hline ergosta-5,22-dien-3-ol & --- & 2.9 & 9.4 & --- & MS \\
\hline ergost-5-en-3 $\beta$-ol & ---- & 3.3 & 9.5 & ---- & MS, co-GC \\
\hline stigmast-5-en-3 $\beta$-ol & --- & 9.8 & 29.6 & --- & MS, co-GC \\
\hline Total & 98.5 & 63.7 & 77.2 & & \\
\hline
\end{tabular}

SAEO: Sinapis alba essential oil

SAET- Sinapis alba EtOH oleoresin SANH- Sinapis alba n-hexane oleoresin

tr: trace $<0.05$

\# the retention index was calculated using a homologous series of $n$-alkanes $\mathrm{C} 8-\mathrm{C} 22$

ФCo-GC: co-injection with an authentic sample.Percentages are the mean of three runs and were obtained from electronic integration measurements using selective mass detector 
The peroxide value of samples containing essential oils were always lesser than the commercial antioxidant BHA but both the oils and oleoresins were not significantly $(p>0.05)$ better antioxidant for linseed oil in comparison to PG. These results have been shown in Figure 1. The secondary oxidation products in linseed oil were measured using thiobarbituric acid value, the same trend was observed for the tested essentials and oleoresins as shown in Figure 2. The stability of linseed oil samples at 200ppm concentration in terms of formation of primary and secondary oxidation process can be ranked in the following descending order:

$P G>B J E O>S A E O>S A E T>B J E T>B H A>B J N H>B J N H$ $>$ SANH

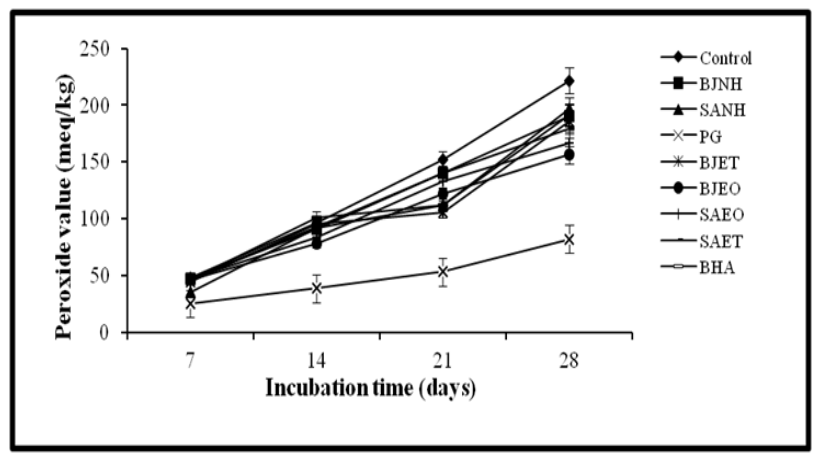

Figure I Antioxidative effect of B.juncea and S.alba essential oils and oleoresins in terms of peroxide value in linseed oil.

Complementary antioxidant assays: The scavenging effect of the B. juncea essential oil (40.2-70.2\%) and oleoresins (21.61-51.78) on DPPH radical linearly increased with increasing concentration whereas the scavenging activity of $S$. alba essential oils and oleoresins ranges in between $19.91-69.77 \%$. The results are plotted in Figure 3. Both essential oils showed moderate to good percent scavenging activity in comparison with BHA at 10, 15 and $20 \mu \mathrm{g}$ level. However, the scavenging ability of PG was best even at $5 \mu \mathrm{g}$.

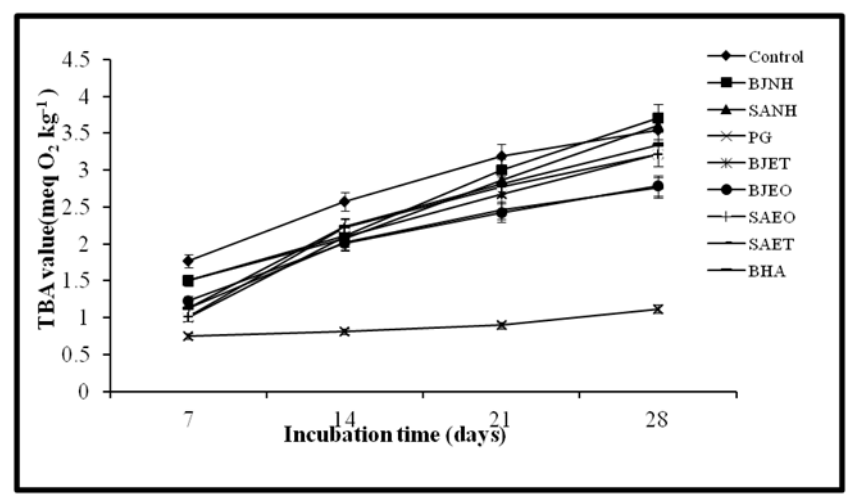

Figure 2 Antioxidative effect of B. juncea and S. alba essential oils and oleoresins in terms of TBA value in linseed oil.

The chelating effect of $B$. juncea essential oil on ferrous ions was $20.25 \%$ at $5 \mu \mathrm{L}$ and increased to $55.15 \%$ at $20 \mu \mathrm{L}$ and the similar trend has been observed for oleoresin (Figure 4). The chelating ability of $S$. alba essential oil on ferrous ions was $20.23 \%$ at $5 \mu \mathrm{L}$ and increased to $53.95 \%$ at $20 \mu \mathrm{L}$ and the similar trend has been observed for oleoresin. However, the chelating ability of EDTA was found to be $96.21 \%$ at $20 \mathrm{mg}$. apparently, the essential oils and oleoresins could chelate ferrous ions but were not as effective chelators as EDTA. The moderate to high chelating effects of essential oils and oleoresins would be beneficial as the ferrous ions are the most effective prooxidants. ${ }^{13}$ The ferric thiocyanate method was used to evaluate the antioxidant potential of essential oils and oleoresins of B.juncea and S.alba and their lipid inhibitory activities were compared with selected standard antioxidants by using. It is believed that high absorbance is an indication of a high concentration of peroxides formed. The absorbance of linoleic acid emulsion without the addition of essential oils or oleoresins or antioxidants increased rapidly. There was a significant $(\mathrm{p}<0.05)$ difference between the blank and the tested essential oils or oleoresins. As can be seen in Figure 5 , the essential oils and oleoresins were able to reduce the formation of peroxides. Higher percentages of allyl isothiocyanates, 1-butene4-isothiocyanate, 5-hydroxymethyl furfural is responsible for the antioxidant actvity of B.juncea and S.alba. ${ }^{3,14}$ These isothiocyanates might be the hydrolysis product of glucosinolates which are water soluble anions. Many workers reported the antioxidant potentials of 5-hydroxymethyl furfural using DPPH scavenging method. ${ }^{15,16}$

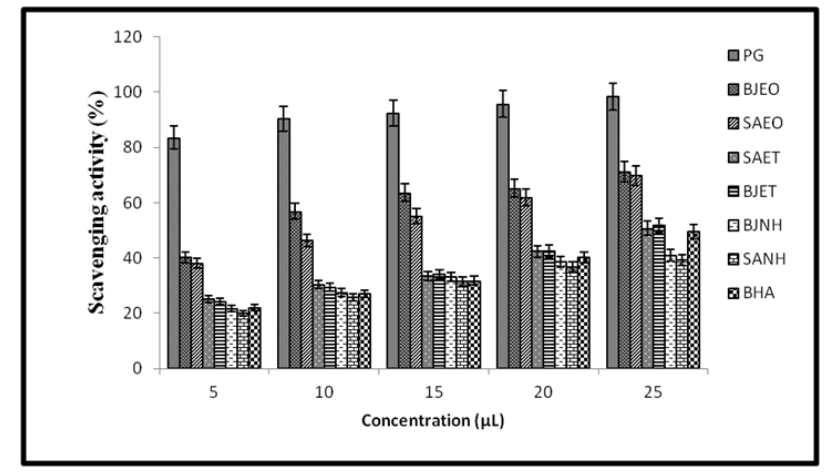

Figure 3 Percent radical scavenging activity of B.juncea and S.alba essential oils and oleoresins on DPPH.

\section{Antimicrobial investigations}

The antifungal activities of B.juncea and S.alba essential oils and oleoresins were investigated using inverted petriplate and food poisoned method. In inverted petriplate method, the essential oils of B.juncea and S.alba exerted considerable zones of growth inhibition (ranges from 45-68\%) against A. niger, P. viridicatum and Fusarium species at all tested doses (Table $3 \mathrm{~A}$ ). The zone of inhibitions showed in Food poisoned method (Table 3B) ranges from $40-60 \%$ for both essential oils against A.niger and Fusarium species whereas for Penicilium viridicatum it is only upto $35 \%$. The oleoresins namely BJET and SAET showed moderate activity against only A.niger (ranges from 20-30\%) in both methods whereas the $\mathrm{n}$ hexane oleoresins did not show any activity. Better activity is obtained at $10 \mu \mathrm{L}$ concentration. The antibacterial activity was investigated using Agar well diffusion method (Table 4). Both essential oils showed strong antibacterial activity against S.aureus and B. subtilis whereas the zone of inhibition recorded for E.coli and P.aeruginosa were very minimal. Zone of inhibition ranges from $11-13 \mathrm{~mm}$ for essential oils is obtained at $10 \mu \mathrm{L}$ concentration. The oleoresins were ineffective at all doses against tested bacteria. The values are found to be statistically significant $(\mathrm{p}<0.05)$ in all these investigations. 
Table 3A Antifungal activity (\% zone of inhibition*) of essential oils and oleoresins of B.juncea and S.alba using inverted plate method.

\begin{tabular}{|c|c|c|c|c|c|c|}
\hline Samples & Doses $(\mu \mathrm{l})$ & $A N^{*}$ & $A F^{*}$ & FM* & FG* & PV* \\
\hline \multirow{2}{*}{ BJEO } & 5 & $43.6 \pm 0.30$ & $47.7 \pm 1.3$ & $39.2 \pm 0.50$ & $39.7 \pm 0.14$ & $34.7 \pm 0.6$ \\
\hline & 10 & $65.9 \pm 0.36$ & $64.3 \pm 1.8$ & $59.7 \pm 0.20$ & $65.7 \pm 0.17$ & $37.6 \pm 0.7$ \\
\hline \multirow{2}{*}{ BJET } & 5 & - & $8.9 \pm 0.20$ & $10 . \pm 0.20$ & - & $9.9 \pm 0.36$ \\
\hline & 10 & $11.2 \pm 0.30$ & $13.2 \pm 0.30$ & $11.3 \pm 0.14$ & $21.5 \pm 2.1$ & $13.7 \pm 0.40$ \\
\hline \multirow{2}{*}{ BJNH } & 5 & - & - & - & - & - \\
\hline & 10 & $5.5 \pm 0.46$ & $7.6 \pm 0.14$ & $4.5 \pm 1.2$ & $9.1 \pm 0.41$ & $5.6 \pm 0.54$ \\
\hline \multirow{2}{*}{ SAEO } & 5 & $29.8 \pm 0.20$ & $21.2 \pm 0.7$ & - & $21.3 \pm 1.5$ & \\
\hline & 10 & $45.2 \pm 0.26$ & $26.4 \pm 3.6$ & $30.1 \pm 2.1$ & $45.6 \pm 1.3$ & $20.9 \pm 0.8$ \\
\hline \multirow{2}{*}{ SAET } & 5 & - & - & - & - & - \\
\hline & 10 & - & - & - & - & - \\
\hline \multirow[t]{2}{*}{ SANH } & 5 & - & - & - & - & - \\
\hline & 10 & - & - & - & - & - \\
\hline
\end{tabular}

Table 3B Antifungal activity (\% zone of inhibition*) of essential oils and oleoresins of B.juncea and S.alba using Food poisoned method.

\begin{tabular}{|c|c|c|c|c|c|c|}
\hline Samples & Doses $(\mu \mathrm{l})$ & $\mathbf{A F}^{*}$ & $\mathrm{AN}^{*}$ & FM* & FG* & PV* \\
\hline \multirow{2}{*}{ BJEO } & 5 & $31.2 \pm 0.50$ & $43.6 \pm 0.30$ & $45.7 \pm 1.3$ & $39.7 \pm 0.14$ & $14.7 \pm 0.6$ \\
\hline & 10 & $39.7 \pm 0.20$ & $60.9 \pm 0.36$ & $60.3 \pm 1.8$ & $59.7 \pm 0.17$ & $17.6 \pm 0.7$ \\
\hline \multirow{2}{*}{ BJET } & 5 & $17.8 \pm 2.4$ & $5.7 \pm 0.20$ & $8.9 \pm 0.20$ & $10 . \pm 0.20$ & - \\
\hline & 10 & $41.9 \pm 0.3$ & $11.2 \pm 0.30$ & $13.2 \pm 0.30$ & $11.3 \pm 0.14$ & - \\
\hline \multirow{2}{*}{ BJNH } & 5 & $54.5 \pm 1.2$ & $0.2 \pm 0.44$ & $4.3 \pm 0.17$ & $2.4 \pm 0.36$ & $5.6 \pm 0.54$ \\
\hline & 10 & $39.8 \pm 0.1$ & $5.5 \pm 0.46$ & $7.6 \pm 0.14$ & $9.1 \pm 0.41$ & $11.7 \pm 0.6$ \\
\hline \multirow{2}{*}{ SAEO } & 5 & $40.1 \pm 2.1$ & $49.8 \pm 0.20$ & $38.2 \pm 0.7$ & $32.6 \pm 1.1$ & $18.1 \pm 0.6$ \\
\hline & 10 & $51.2 \pm 2.3$ & $55.2 \pm 0.26$ & $56.4 \pm 3.6$ & $57.1 \pm 1.7$ & $20.9 \pm 0.8$ \\
\hline \multirow{2}{*}{ SAET } & 5 & - & - & - & - & - \\
\hline & 10 & - & - & - & - & - \\
\hline \multirow{2}{*}{ SANH } & 5 & - & - & - & - & - \\
\hline & 10 & $17.8 \pm 1.2$ & $15.6 \pm 1.5$ & $11.2 \pm 1.7$ & $16.7 \pm 1.2$ & - \\
\hline
\end{tabular}

Table 4 Antibacterial activity of B.juncea and S.alba essential oils and its oleoresins against a few bacterial species using agar well diffusion method

\begin{tabular}{llllll}
\hline Samples & Doses $(\mathbf{p p m})$ & BS & SA & EC & PA \\
\hline \multirow{2}{*}{ BJEO } & 1000 & $10.2 \pm 1.5$ & $11.8 \pm 1.7$ & 3.61 .8 & $4.7 \pm 1.3$ \\
\multirow{2}{*}{ SAEO } & 3000 & $13.6 \pm 1.3$ & $12.3 \pm 2.3$ & $4.5 \pm 1.6$ & $5.9 \pm 1.5$ \\
& 1000 & $11.3 \pm 0.9$ & $14.3 \pm 1.4$ & $4.4 \pm 1.4$ & $3.4 \pm 1.7$ \\
BJET & 3000 & $12.4 \pm 0.6$ & $15.7 \pm 0.3$ & $5.7 \pm 1.2$ & $4.9 \pm 1.1$ \\
\multirow{2}{*}{ SAET } & 1000 & - & - & - & - \\
& 3000 & - & - & - & - \\
BJNH & 1000 & - & - & - & - \\
& 3000 & - & - & - & - \\
SANH & 1000 & - & - & - & - \\
\multirow{2}{*}{ Ampicillin } & 3000 & - & - & - & - \\
& 1000 & $13.3 \pm 2.1$ & $15.4 \pm 0.9$ & - & $11.6 \pm 1.3$ \\
\hline
\end{tabular}

Diameter of inhibition zone $(\mathrm{mm \#})$ 
The results found in above investigation were well correlated with the previous work. ${ }^{17-20}$ The chemical composition revealed that isothiocyanates are the major components in both essential oils. Isothiocyanates arise in plants as a result of enzymatic cleavage of released glucosinolates from intracellular compartments by membranebound myrosinase upon damage to the plant. ${ }^{21}$ Myrosinase promotes hydrolysis and intramolecular rearrangement of intermediates, resulting in the three main groups of substances: nitriles, thiocyanates, and isothiocyanates. ${ }^{22}$ Allyl isothiocyanate (AITC) in vapor and liquid forms has demonstrated high bactericidal activity against various food spoilage microorganisms and food pathogens. ${ }^{23}$ Nielsen et al. ${ }^{24}$ showed the broad spectrum activity of allyl isothiocyanates against many fungi. The structure of isothiocyanates is responsible for their efficacy: the more volatile the compound, the greater its antibiological activity due to better distribution. The type of microorganisms being combated, and even the particular phase of their growth, is also important. ${ }^{25}$ The mode of action behind AITC's antimicrobial activity is not yet fully understood, but since it might penetrate membranes and no single site of action has been described, it is generally regarded as a non-specific inhibitor of periplasmic or intracellular targets. The biocidal activity of isothiocyanates is comparable with the efficacy of synthetic pesticides, like methyl bromide, and some antibiotics (gentamycin). ${ }^{26}$ It has been already established that essential oils and oleoresins are complex mixtures of components that show usually higher activities than their isolated components; their final activities are due to the combined effects of several minor components. Thus, they contain multifunctional components that exert their activities through different mechanisms. ${ }^{27}$

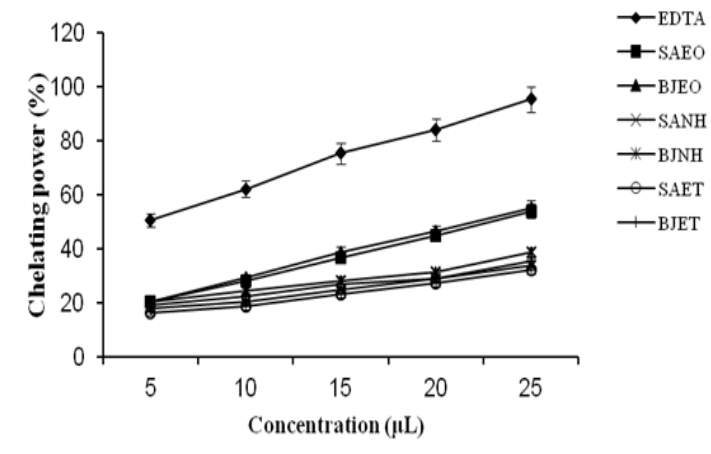

Figure 4 Percent chelating power of B.juncea and S.alba essential oils and oleoresins.

It has been demonstrated that the antimicrobial effects of the essential oils acts by causing structural and functional damages to the bacterial cell membrane. It is also indicated that the optimum range of hydrophobicity is involved in the toxicity of the essential oils. Spices and herbs are mostly used in the range of $0.05-0.1 \%$ $(500-1000 \mathrm{ppm})$ in food systems. Some spices have stronger antimicrobial activity than others and can be effective at 1000ppm. However, some spices require higher concentrations..$^{28}$ Application of antimicrobials by different exposure methods, such as vapor phase compared to direct contact method, of mustard and clove essentials oils showed noteworthy differences. ${ }^{29}$ The stereochemistry, lipophilicity and other factors affected the biological activity of these compounds which might be altered positively or negatively by slight modifications. ${ }^{30}$ It has been shown that plant substances affect microbial cells by various antimicrobial mechanisms, including attacking the phospholipid bilayer of the cell membrane, disrupting enzyme systems, compromising the genetic material of bacteria, and forming fatty acid hydroperoxidase caused by oxygenation of unsaturated fatty acids. ${ }^{31}$ Allyl isothiocyanate derived from mustard seems to have multi-targeted mechanisms of action in metabolic pathways generally; Gram-negative bacteria are less sensitive to the antimicrobials because of the lipopolysaccharide outer membrane of this group, which restricts diffusion of hydrophobic compounds. However, this does not mean that Gram-positive bacteria are always more susceptible. Gram-negative bacteria are usually more resistant to the plant-origin antimicrobials and even show no effect, compared to Gram-positive bacteria. ${ }^{32}$

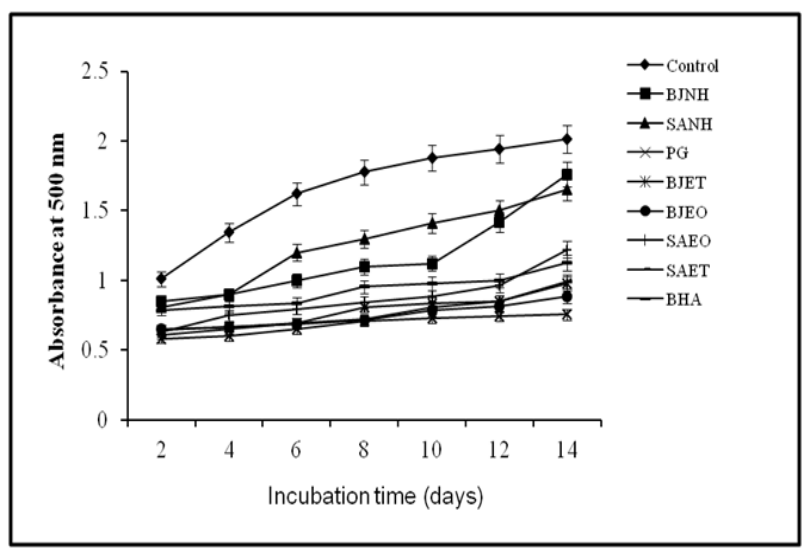

Figure 5 Inhibitory effect on primary oxidation using FTC method of B.juncea and S.alba essential oils and oleoresins.

\section{Conclusion}

As a conclusion all findings reported here that B.juncea and S.alba essential oils may be selected for the use as potentially food preservatives in fermented products and other foods, depending upon the desired flavor of the products.. This study is an attempt to gain a better understanding of the chemical profiles of the essential oils and oleoresins of Brassicaceae members. Therefore, it is of interest to proceed with research dealing with the pharmaceutical applications for the consumers.

\section{Acknowledgements}

We acknowledge profound gratitude to Head, Chemistry Department Deen Dayal Upadhyaya Gorakhpur University, Gorakhpur, India for providing laboratory facilities. The authors also wish to thanks the University Grants Commission and Department of Science \& Technology for providing financial assistance to Sunita Singh (UGC-SRF) and Dr. Gurdip Singh (Emeritus Scientist) respectively.

\section{Conflict of interest}

The author declares no conflict of interest.

\section{References}

1. Podsedek A. Natural antioxidants and antioxidant capacity of Brassica vegetables: A review. Lwt Food Sci Technol. 2007;40(1):1-11.

2. Abbasi BH, Rashid A, Khan MA, et al. In vitro plant regeneration in sinapis alba and evaluation of its radical scavenging activity. Pak J of Bot. 2011;43:21-27. 
3. Drewnowski A, Gomez-Carneros C. Bitter taste, phytonutrients, and the consumer: A review. Am J Clin Nutr. 2000;72(6):1424-1435.

4. Massoneuve SA, Ruffine S. European Pharmacopoeia. 1983;1:4-8.

5. Adams RP. Identification of essential oil components by Gas Chromatography/Mass Spectrometry. Illinois, USA: Allured Publishing Corp; 2001.

6. Bandoniene D, Gruzdiene D, Venskutonis PR. Antioxidant activity of sage extracts in rapeseed oil irradiated with UV rays. Nahrung. 2001;45(2):105-108.

7. Singh G, Maurya S, Lampasona MP, et al. Chemical constituents, antimicrobial investigations and antioxidative potentials of Anethum graveolens L. Essential oil and acetone extract: Part 52. J Food Sci.2005;70(4):M208-M215.

8. Singh G, Kapoor IPS, Singh P, et al. Chemistry, antioxidant and antimicrobial investigations on essential oil and oleoresins of Zingiber officinale. Food Chem Toxic. 2008;46(10):3295-3302.

9. Alvarez-Castellanos PP, Bishop CD, Pascual-Villalobos MJ. Antifungal activity of the essential oil of flowerheads of garland chrysanthemum (Chrysanthemum coronarium) against agricultural pathogens. Phytochemistry. 2001;57(1):99-102.

10. Ramdas K, Suresh G, Janardhanan N, et al. Antifungal activity of 1,3-disubstituted symmetrical and unsymmetrical Thioureas. Pesticidal Sci. 1998;52(2):145-151.

11. Wittstock U, Halkier BA. Glucosinolate research in the Arabidopsis era. Trends Plant Sci. 2002;7(6):263-270.

12. Joardar A, Das S. Effect of fatty acids isolated from edible oils like mustard, linseed or coconut on astrocytes maturation. Cell Mol Neurobiol. 2007;27(8):973-983.

13. Yamaguchi R, Tatsumi MA, Kato K, et al. Effect of metal salts and fructose on the autoxidation of methyl linoleate in emulsion. Agric Biologicol Chemistry. 1998;52(3):849-850.

14. Jahangir M, Kim HK, Choi YH, et al. Health affecting compounds in Brassicaceae. Comp Rev Food Sci Food Safety. 2009;8(2):31-43.

15. Liua Z, Liub Y, Chaoc Z, et al. Nat Prod Comm. 2011;6(1):55.

16. Singhara A, Macku C, Shibamoto T. Antioxidative Activity of Brewed Coffee Extracts. Functional Foods for Disease Prevention II American Chemical Society. Washington, USA; 1998;702(11):101-109.

17. Blazevic I, Radonic A, Mastelic J, et al. Glucosinolates, glycosidically bound volatiles and antimicrobial activity of Aurinia sinuata (Brassicaceae) Food Chem. 2010;121(4):1020-1028.

18. Szczyglowska M, Piekarska A, Konieczka P, et al. Use of brassica plants in the phytoremediation and biofumigation processes. Int $\mathrm{J} \mathrm{Mol} \mathrm{Sci}$. 2011;12(11):7760-7771.
19. Shin SW, Kang CA. Studies on compositions and antifungal activities of essential oils from cultivars of Brassica juncea L. Korean Journal of Pharmacognosy. 2001;32:140-144.

20. Lazzeri L, Leoni O, Manici L. Biocidal plant dried pellets for biofumigation. Ind Crops ProdInd Crops Prod. 2004;20(1):59-65.

21. Delaquis P, Sholberg PL. Antimicrobial activity of gaseous ally isothiocyanate. J Food Prot. 1997;60(8):943-957.

22. Zhang Y, Talalay P. Anticarcinogenic activities of organic isothiocyanates: chemistry and mechanisms. Cancer Res. 1994;54(7suppl):1976s-1981.

23. Luciano FB, Holley RA. Enzymatic inhibition by allyl isothiocyanate and factors affecting its antimicrobial action against Escherichia coli O157:H7. Int J Food Microbiol. 2009;131(2-3):240-245.

24. Nielsen, PV, Rios R. Inhibition of fungal growth on bread by volatile components from spices and herbs, and the possible application in active packaging, with special emphasis on mustard essential oil. Int $J$ Food Microbiol. 2003;60(2-3):219-229.

25. Gimsing AL, Kirkegaard JA. Glucosinolates and biofumigation: fate of glucosinolates and their hydrolysis products in soil. Phytochem Rev. 2009;8:299.

26. Lugauskas A, Repeckiene J, Uselis N, et al. A Problems on a longtime strawberry growing in one plot. Acta Polonorum Scientarium Hortorum Cultus. 2003;2(2):59-68.

27. Lord J, Lazzeri, L, Atkinson H, et al. Biofumigation for control of pale potato cyst nematodes: Activity of Brassica leaf extracts and green manures on Globodera pallida in vitro and in soil. J Agric Food Chem. 2011;59(14):7882-7890.

28. Ceylan E, Fung, DYC. Antimicrobial activity of spices. J Rapid Methods Automat Microbiol. 2004;12(1):1-55.

29. Goni P, Lopez P, Sanchez C, et al. Antimicrobial activity in the vapour phase of a combination of cinnamon and clove essential oils. Food Chemistry. 2009;116(4):982-989.

30. Veluri R, Weir TL, Bais HP, et al. Phytotoxic and antimicrobial activities of catechin derivatives. J Agric Food Chem. 2004;52(5):1077-1082.

31. Skocibusic M, Bezic N, Dunkic V. Phytochemical composition and antimicrobial activities of the essential oils from Satureja subspicata Vis. growing in Croatia. Food Chemistry. 2006;96(1):20-28.

32. Stefanello MEA, Cervi A C, Ito IY, et al. Chemical composition and antimicrobial activity of essential oils of Eugenia chlorophylla (Myrtaceae). J Essen Oil Res. 2008;20(1):75-78. 